\title{
Successful Resection in a Case of Hepatocellular Carcinoma Masquerading as Hilar Cholangiocarcinoma: A Case Report
}

\author{
Prakriti Gupta $^{1}$, Shashi Dhawan ${ }^{1}$, Vivek Mangla*2 ${ }^{2}$ Siddharth Mehrotra ${ }^{2}$, Shailendra Lalwani ${ }^{2}$, \\ Naimish N Mehta ${ }^{2}$ and Samiran Nundy ${ }^{2}$ \\ ${ }^{1}$ Department of Pathology, Sir Ganga Ram Hospital, New Delhi, India; ${ }^{2}$ Department of Surgical Gastroenterology and Liver \\ Transplantation, Sir Ganga Ram Hospital, New Delhi, India
}

\begin{abstract}
Obstructive jaundice in the setting of a hilar mass raises strong suspicion of a cholangiocarcinoma, with diagnosis usually made on imaging. Jaundice, when present in patients with hepatocellular carcinoma, is mostly related to underlying liver disease (i.e. cirrhosis) and only rarely to diffuse tumor infiltration of liver parenchyma or hilar invasion. We report here the good outcome of a 67 year-old patient who presented with obstructive jaundice, underwent surgery and was given a diagnosis of hepatocellular carcinoma, which was made only at histopathological examination of resected specimen.

Citation of this article: Gupta P, Dhawan S, Mangla V, Mehrotra S, Lalwani S, Mehta NN, et al. Successful resection in a case of hepatocellular carcinoma masquerading as hilar cholangiocarcinoma: A case report. J Clin Transl Hepatol 2018;6(4):442446. doi: $10.14218 / \mathrm{JCTH} .2018 .00038$.
\end{abstract}

\section{Introduction}

Obstructive jaundice in the setting of a hilar mass raises strong suspicion of a cholangiocarcinoma. The diagnosis is usually made on imaging, and cytology confirmation is usually not done for tumors amenable to surgical resection. Jaundice at the time of diagnosis is seen in a small proportion of patients with hepatocellular carcinoma (HCC) and is mostly related to underlying liver disease (i.e. cirrhosis) and only rarely to diffuse tumor infiltration of liver parenchyma or hilar invasion. ${ }^{1}$ There have been only a few and scattered reports in the literature of such presentations of HCC, with no available report of resection in such a patient to the best of our knowledge. ${ }^{2-11}$ We report herein the good outcome of an unusual case of $\mathrm{HCC}$ presenting as obstructive jaundice and diagnosed initially as hilar cholangiocarcinoma, which was treated with surgery.

Keywords: Obstructive jaundice; Hepatocellular carcinoma; Cholangiocarcinoma. Abbreviations: AFP, alpha fetoprotein; CA19-9, carbohydrate antigen 19-9; HCC hepatocellular carcinoma; IHC, immunohistochemistry; PTBD, percutaneous transhepatic biliary drainage.

Received: 12 June 2018; Revised: 27 July 2018; Accepted: 13 August 2018

*Correspondence to: Vivek Mangla, Department of Surgical Gastroenterology and Liver Transplantation, Sir Ganga Ram Hospital, Room No. 2222, 2nd Floor, SSRB, Old Rajender Nagar, New Delhi 110060, India. Tel: +91-9868352888, Fax: +91-11-25861002, E-mail: mangla.vivek@gmail.com
Case report

A 67 year-old male (body mass index of $23.9 \mathrm{~kg} / \mathrm{m}^{2}$ ) presented with jaundice associated with pruritus for 1 month. There was no history of abdominal pain or weight loss. There was no previous history of regular alcohol intake, jaundice, ascites, gastrointestinal bleeding, chronic illnesses or medication intake, surgery or hepatic encephalopathy. Liver function tests revealed a total bilirubin of 12.8 (normal range: $0.2-1$ ) $\mathrm{mg} / \mathrm{dL}$, with elevated liver enzymes and normal serum albumin (Table 1). Prothrombin time and platelet counts were normal.

Ultrasound of the abdomen revealed intrahepatic biliary radical dilatation with possibility of a mass present at the

Table 1. Demographics and laboratory parameters of the patient

\begin{tabular}{ll}
\hline Parameter & Value \\
\hline Age & 67 years \\
Sex & Male \\
Weight & $70 \mathrm{~kg}$ \\
Height & $171 \mathrm{~cm}$ \\
BMI & $23.9 \mathrm{~kg} / \mathrm{m}^{2}$ \\
Platelet count & $1.7 \times 10^{5} / \mathrm{cubic} \mathrm{mL}$ \\
International normalized & 1.1 \\
ratio (PT-INR) & Negative \\
HBsAg & Negative \\
Anti-HCV & $12.8 / 8.9 \mathrm{mg} / \mathrm{dL}$ \\
Bilirubin at first presentation, & \\
total/direct & $98 \mathrm{IU} / \mathrm{L}$ \\
AST & $106 \mathrm{IU} / \mathrm{L}$ \\
ALT & $360 \mathrm{IU} / \mathrm{L}$ \\
Serum alkaline phosphatase & $100 \mathrm{IU} / \mathrm{L}$ \\
Gamma-glutamyltransferase & $3.7 \mathrm{~g} / \mathrm{dL}$ \\
Serum albumin & $3.9 / 2.6 \mathrm{mg} / \mathrm{dL}$ \\
Bilirubin just prior to surgery, & $1996 \mathrm{U} / \mathrm{mL}$ \\
total/direct & \\
CA19-9 & Al \\
\hline
\end{tabular}

Abbreviations: Anti-HCV, hepatitis C virus antibodies; ALT, alanine transaminase; AST, aspartate transaminase; BMI, body mass index; CA19-9, carbohydrate antigen 19-9; HBsAg, hepatitis B surface antigen. 


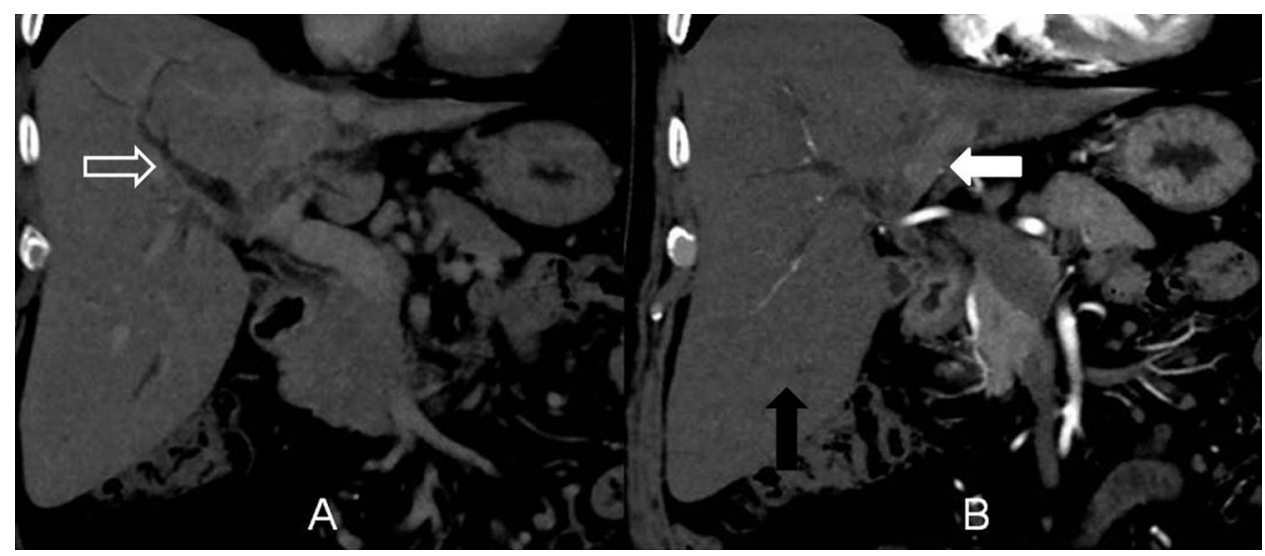

Fig. 1. Portal venous phase images showing dilatation of right-sided intrahepatic bile ducts (hollow white arrow), atrophy of left hemiliver and hypertrophy of right hemiliver (solid black arrow). (A) The arterial phase images show the mass lesion (solid white arrow) and free right hepatic artery (B).

hepatic hilum. Computed tomography scan revealed a heterogeneously enhancing mass lesion involving the left and common hepatic ducts, with encasement of left portal vein and left hepatic artery. There was significant hypertrophy of the right liver and atrophy of the left hemiliver. The right and main portal vein, and the right hepatic artery were free of tumor (Fig. 1). There was no evidence of ascites or splenomegaly. Carbohydrate antigen 19-9 (CA19-9) was 1996 (normal range, 0-35) $\mathrm{U} / \mathrm{mL}$. Tests for Hepatitis B surface antigen and anti-hepatitis $C$ virus antibodies were negative (Table 1). A provisional diagnosis of hilar cholangiocarcinoma was made.

In view of a planned major liver resection and significantly elevated serum bilirubin, preoperative percutaneous transhepatic biliary drainage (PTBD) was carried out to drain the right posterior sectoral duct. Following PTBD, the patient's bilirubin decreased to $3.9 \mathrm{mg} / \mathrm{dL}$ over the next 15 days. At surgery, the computed tomography findings were confirmed. The liver was firm but there was no evidence of cirrhosis. There was also no evidence of dissemination. The right

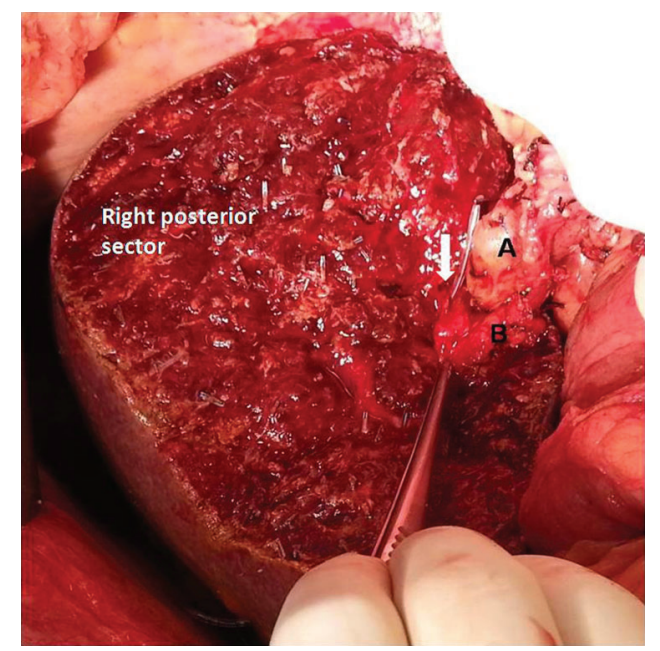

Fig. 2. Intraoperative photograph taken after complete resection shows the bile duct of the liver remnant with a feeding tube put through the PTBD tract (solid white arrow), main portal vein (A), and right hepatic artery (B). hepatic artery, right portal vein and main portal vein were free. The patient underwent extended left hepatectomy with excision of caudate lobe and extrahepatic biliary tree, with hepaticojejunostomy to the remnant bile duct (Figs. 2, 3). There was no evidence of tumor infiltration in the proximal or distal bile duct margins on frozen section analysis carried out intraoperatively.

Gross examination of the hepatectomy specimen revealed a slightly nodular surface. On serial slicing, there was an intraluminal mass of $2 \times 1.5 \mathrm{~cm}$ in the common hepatic duct along with a diffusely infiltrating grey white tumor mass in the hilar region. A conglomeration of small tumor nodules was also seen in the subcapsular region, approximately $4 \mathrm{~cm}$ from the main tumor mass in the hilar region. On further slicing, the tumor was seen to infiltrate the intrahepatic biliary system, extending from the hilar to the subcapsular region (Fig. 4). On microscopic examination, there was an intraluminal mass found in the common hepatic duct with surface ulceration and necrosis, infiltrating the intrahepatic biliary ducts. The tumor cells were large polygonal, with eosinophilic cytoplasm and moderate nuclear atypia, arranged in cords, trabeculae and sheets. There was no evidence of vascular invasion. All the surgical margins were free of tumor (R0 resection). The

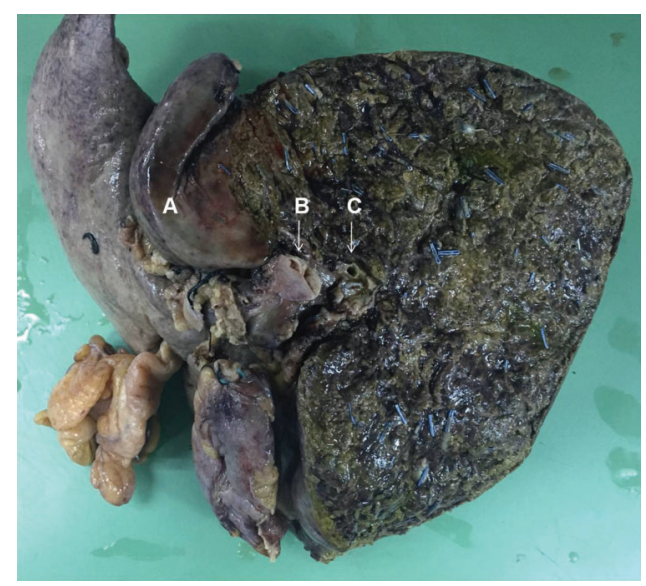

Fig. 3. Photograph of resected specimen showing the caudate lobe (A), distal bile duct (B) and right posterior sectoral duct margin (C). 


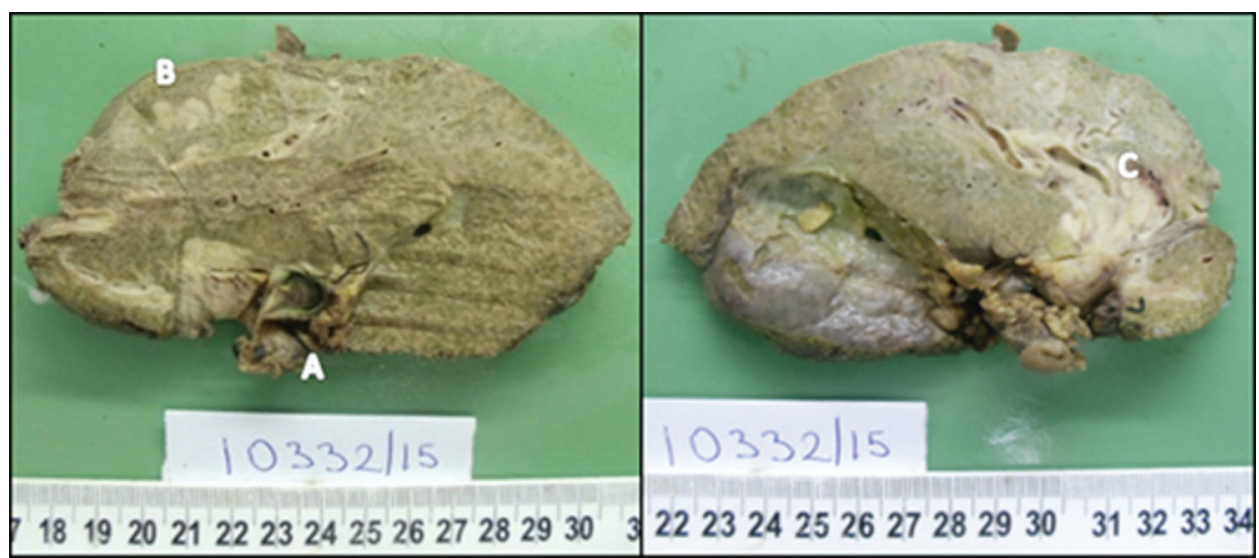

Fig. 4. Gross examination findings included intraluminal mass in the common hepatic duct (A), subcapsular liver nodules (B), and intrahepatic bile ducts infiltrated by the tumor (C).

surrounding liver parenchyma showed nodularity and portal fibrosis with focal bridging fibrosis (Fig. 5). Immunohistochemistry (IHC) revealed strong and diffuse Hep par 1 positivity in the tumor cells in both the common hepatic duct mass and hepatic nodules. Glypican 3 was focally positive in both the tumor masses (Fig. 6). CK7 and MUC-1 were negative in the tumor cells. Based upon the morphology and IHC, a final diagnosis of HCC (TNM stage, pT2 pNO pMx) with extensive infiltration of biliary system was made.

The postoperative course was marked by transient ascites, which improved with medical treatment. The patient was discharged on postoperative day 12 . At 3 years follow-up, the patient was well, with normal liver function tests and no evidence of disease recurrence or liver decompensation.
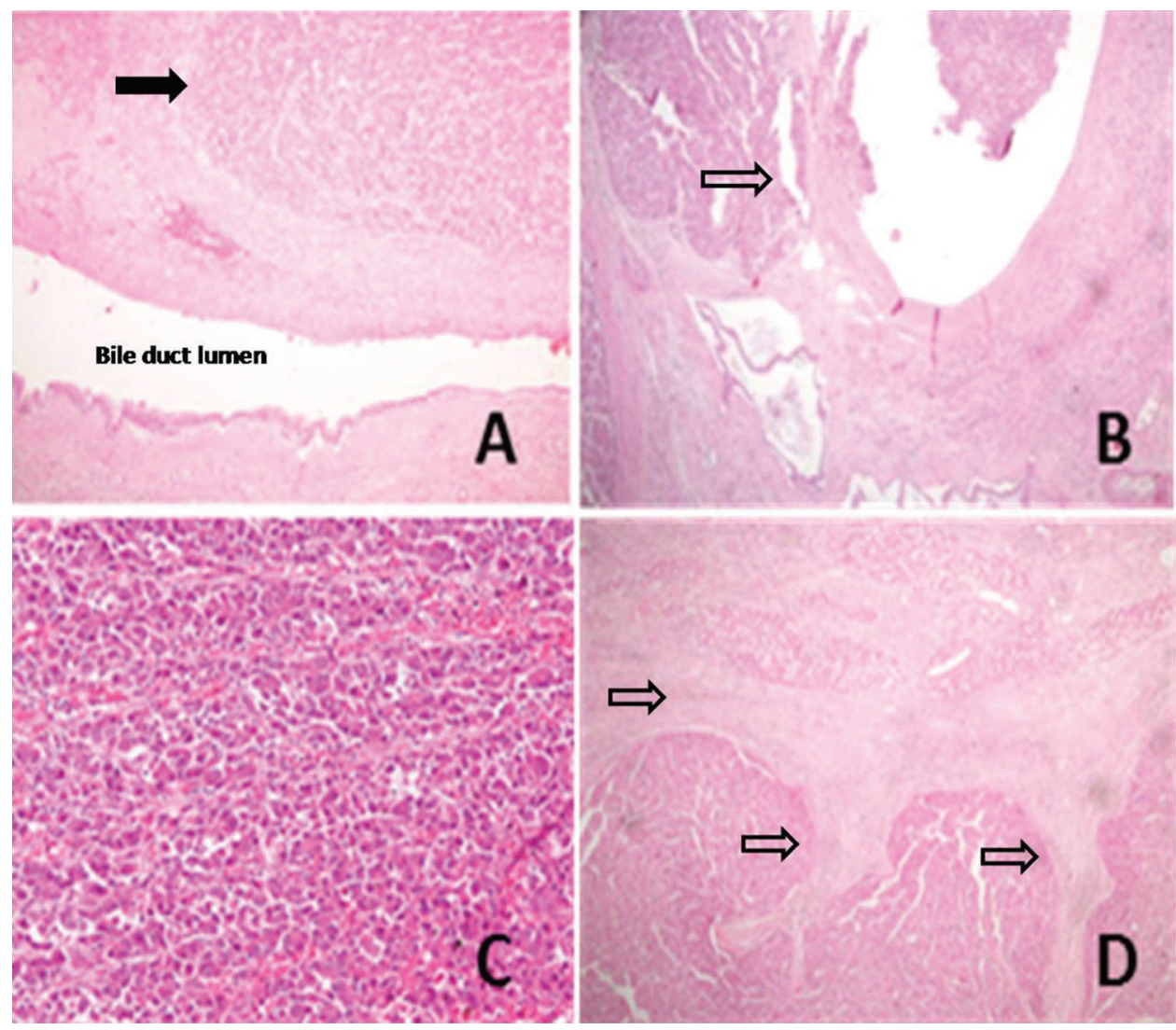

Fig. 5. Microscopic examination findings. Hematoxylin-eosin (HE) staining, showing: tumor infiltrating common hepatic duct wall (solid black arrow), HE 40X ( infiltration into the intrahepatic bile ducts (hollow arrows), HE 40X (B); polygonal tumor cells in a trabecular arrangement, HE 400X (C); and, bridging fibrosis (hollow arrows), HE 40X (D). 


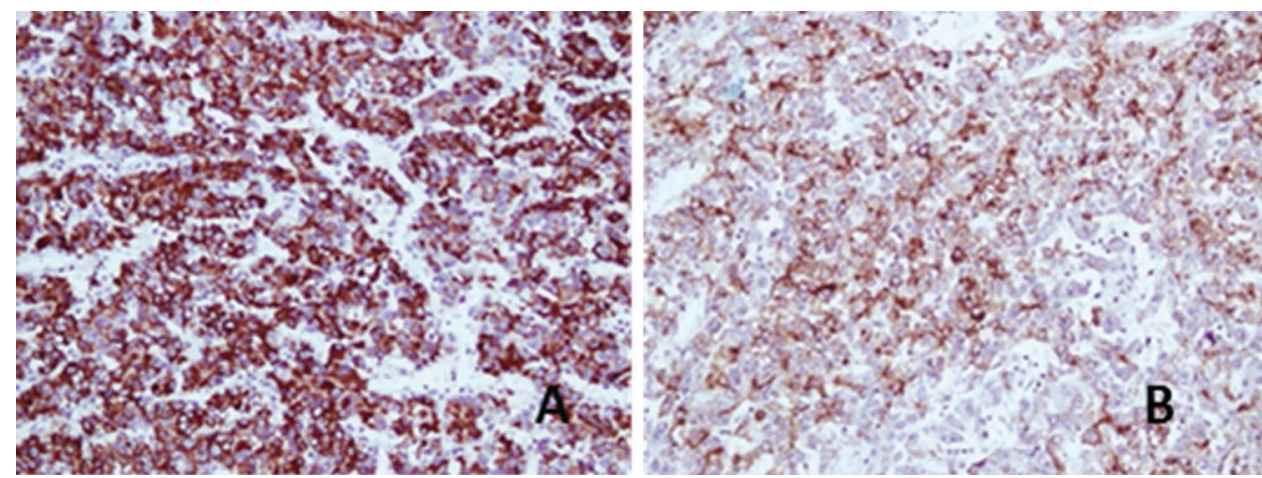

Fig. 6. Immunohistochemistry showing a strong and diffuse cytoplasmic positivity with Hep Par 1 (A) and Glypican 3 (B), $200 x$. The tumor cells were negative for CK7 and MUC-1.

\section{Discussion}

Obstructive jaundice in the setting of a hilar mass raises strong suspicion of cholangiocarcinoma. In patients with hilar cholangiocarcinoma, the diagnosis is usually made on imaging, and cytology confirmation is usually not mandatory for tumors amenable to surgical resection. Although there are imaging criteria which help differentiate intrahepatic cholangiocarcinoma from HCC, no differentiating features have been reported on imaging for differentiating between hilar cholangiocarcinoma and $\mathrm{HCC}$ infiltrating the bile ducts. ${ }^{12}$ Tumor markers are also, often, of only limited help. Alpha fetoprotein (AFP) levels are not elevated in a significant proportion of patients with HCC. ${ }^{13,14}$ CA19-9 is usually elevated in patients with cholangiocarcinoma but may be falsely elevated in certain situations (e.g., cholangitis, biliary obstruction, smokers, etc.). The diagnosis in previously reported cases of HCC masquerading as hilar cholangiocarcinoma was suspected on imaging, presence of elevated AFP levels, presence of coexisting viral infections (i.e. Hepatitis B or C) or nonimprovement of bilirubin despite PTBD (arousing the suspicion of underlying liver disease). The initial diagnosis of hilar cholangiocarcinoma in our patient was based on computed tomography findings and an elevated CA19-9. AFP was not measured in our case, as the possibility of HCC was not considered.

HCC usually occurs on the background of a cirrhotic liver. Patients with underlying cirrhosis are usually not candidates for major liver resection and, probably, that is why most reported cases in the literature were deemed unresectable. ${ }^{15}$ However, we feel that surgical resection can be considered in patients who are good candidates for surgery (i.e. those with preserved liver functions, good performance status, and resectable tumors with adequate future liver remnant). The overall survival of patients with HCC with obstructive jaundice is much better than those with jaundice due to hepatic insufficiency, for obvious reasons. ${ }^{16}$

IHC has an important role in differentiating between HCC, cholangiocarcinoma and mixed HCC-cholangiocarcinoma. Tumor cells in cholangiocarcinoma are typically positive for CK7 and MUC1 and negative for Hep par 1 and Glypican 3. Most cases of HCC will have tumor cell positivity for Hep par 1 and Glypican 3 and would be negative for CK7 and MUC1. The final diagnosis of HCC was confirmed on IHC in our patient. The mixed HCC and cholangiocarcinoma would have tumor cells expressing markers for both HCC and cholangiocarcinoma.
The present case had a normal platelet count and prothrombin time, no evidence of portal hypertension on imaging, a rapid improvement in jaundice with PTBD and a good future liver remnant. This enabled a major liver resection with good outcomes. There was nothing to suggest the presence of underlying liver disease on preoperative evaluation, in imaging, or even at surgery. It was only at final histopathology that the diagnosis of HCC and underlying liver disease could be made.

To the best of our knowledge, this is the first such report of a case of HCC masquerading as a hilar cholangiocarcinoma to have a surgical resection with good long-term outcomes.

\section{Conclusions}

HCC infiltrating the biliary system may sometimes masquerade as hilar cholangiocarcinoma. Good outcomes can be achieved with complete resection in patients with preserved liver functions and absence of portal hypertension

\section{Conflict of interest}

The authors have no conflict of interests related to this publication.

\section{Author contributions}

Writing of the first draft and provision of material support (PG), provision of material support and critical revision of the drafted manuscript (SD), performance of the surgery, provision of material support and critical revision of the drafted manuscript (VM), critical revision of the drafted manuscript and provision of funding (SM, SL, NNM, SN).

\section{References}

[1] Kew MC, Paterson AC. Unusual clinical presentations of hepatocellular carcinoma. Trop Gastroenterol 1985;6:10-22. doi: 10.1155/2000/79241.

[2] Lau WY, Leow CK, Leung KL, Leung TW, Chan M, Yu SC. Cholangiographic features in the diagnosis and management of obstructive icteric type hepatocellular carcinoma. HPB Surg 2000;11:299-306. doi: 10.1155/ 2000/79241.

[3] Mallory TB, Castleman B, Parris EE. Case records of the Massachusetts General Hospital. N Eng J Med 1947;237:667-673.

[4] Kojiro M, Kawabata K, Kawano Y, Shirai F, Takemoto N, Nakashima T. Hepatocellular carcinoma presenting as intrabile duct tumor growth: a clinicopathologic study of 24 cases. Cancer 1982;49:2144-2147. 
doi: $10.1002 / 1097-0142(19820515) 49: 10 \leq 2144:: A I D-C N C R 2820491026 \geq 3$. $0 . \mathrm{CO} ; 2-\mathrm{O}$.

[5] Ishikawa I, Kobayashi K, Odajima S, Takada A, Takeuchi J. Primary hepatic cancer with recurrent episodes of obstructive jaundice and distended gallbladder. A case study and review of the litterature. Am J Gastroenterol 1973; 60:496-503.

[6] Ihde DC, Sherlock P, Winawer SJ, Fortner JG. Clinical manifestations of hepatoma. A review of 6 years' experience at a cancer hospital. Am J Med 1974; 56:83-91. doi: 10.1016/0002-9343(74)90753-0.

[7] Roslyn JJ, Kuchenbecker S, Longmire WP, Tompkins RK. Floating tumor debris. A cause of intermittent biliary obstruction. Arch Surg 1984;119: 1312-1315. doi: 10.1001/archsurg.1984.01390230078019.

[8] Okuda K, Kubo Y, Okazaki N, Arishima T, Hashimoto M. Clinical aspects of intrahepatic bile duct carcinoma including hilar carcinoma: a study of 57 autopsy-proven cases. Cancer 1977;39:232-246. doi: 10.1002/1097-0142 (197701)39:1<232::AID-CNCR2820390137>3.0.CO;2-Y.

[9] Wang JH, Wang LY, Lin ZY, Chen SC, Kang SC, Chuang WL, et al. Doppler sonography of common hepatic duct tumor invasion in hepatocellular carcinoma: report of two cases. J Ultrasound Med 1995;14:471-474. doi: 10 7863/jum.1995.14.6.471.

[10] Jurco S 3rd, Kim HS. Extrahepatic biliary obstruction by hepatocellular carcinoma. Am J Gastroenterol 1980;74:176-178.
[11] Terada T, Nakanuma Y, Kawai K. Small hepatocellular carcinoma presenting as intrabiliary pedunculated polyp and obstructive jaundice. J Clin Gastroenterol 1989;11:578-583. doi: 10.1097/00004836-19891000000019.

[12] Wengert GJ, Baltzer PAT, Bickel H, Thurner P, Breitenseher J, Lazar M, et al. Differentiation of intrahepatic cholangiocellular carcinoma from hepatocellular carcinoma in the cirrhotic liver using contrast-enhanced MR imaging. Acad Radiol 2017;24:1491-1500. doi: 10.1016/j.acra.2017.06.005.

[13] Carr BI, Akkiz H, Üsküdar O, Yalçõn K, Guerra V, Kuran S, et al. HCC with lowand normal-serum alpha-fetoprotein levels. Clin Pract (Lond) 2018;15:453464. doi: $10.4172 /$ clinical-practice. 1000393.

[14] Chan SL, Mo F, Johnson PJ, Siu DY, Chan MH, Lau WY, et al. Performance of serum $\alpha$-fetoprotein levels in the diagnosis of hepatocellular carcinoma in patients with a hepatic mass. HPB (Oxford) 2014;16:366-372. doi: 10 . $1111 / \mathrm{hpb} .12146$.

[15] Ise N, Andoh $\mathrm{H}$, Sato T, Yasui $O$, Kurokawa $T$, Kotanagi $H$. Three cases of small hepatocellular carcinoma presenting as obstructive jaundice. HPB (Oxford) 2004;6:21-24. doi: 10.1080/13651820310017129.

[16] Lau W, Leung K, Leung TW, Liew CT, Chan MS, Yu SC, et al. A logical approach to hepatocellular carcinoma presenting with jaundice. Ann Surg 1997;225: 281-285. doi: 10.1097/00000658-199703000-00007. 